\title{
ENTORNO VIRTUAL COMO SOPORTE A LA FORMACIÓN DE INFORMÁTICOS EN UNA UNIVERSIDAD DEL NORDESTE ARGENTINO
}

\section{VIRTUAL ENVIRONMENT TO SUPPORT COMPUTER TRAINING AT A UNIVERSITY IN NORTHEASTERN ARGENTINA}

\author{
María Victoria López; vlopez@arnet.com.ar \\ Gladys N. Dapozo; lalydapozo@hotmail.com \\ Cristina L. Greiner ; cgreiner33@hotmail.com \\ María C. Espíndola; mcespindola@yahoo.com \\ Universidad Nacional del Nordeste (Argentina)
}

\section{RESUMEN}

Se presenta una metodología de enseñanza que utiliza un entorno virtual como apoyo a las actividades presenciales de la asignatura Algoritmos y Estructuras de Datos I de la carrera Licenciatura en Sistemas de Información de la Universidad Nacional del Nordeste en Argentina, cuyo objetivo es contribuir a la organización de las actividades y la comunicación con los alumnos, además de aportar a su formación disciplinar específica.

PALABRAS CLAVE: Entorno virtual, blended learning, formación universitaria en Informática.

\begin{abstract}
It presents a teaching methodology that uses a virtual environment to support classroom activities of the subject "Algorithms and Data Structures I" of the career of Information Systems from the Universidad National del Nordeste in Argentina, which aims to contribute to the organization activities and communication with students, besides providing training to its specific discipline.
\end{abstract}

KEYWORDS: Virtual environment, blended learning, university degree in Informatics. 


\section{INTRODUCCIÓN}

Las Tecnologías de la Información y la Comunicación (TIC) actualmente se insertan en todas las áreas de la sociedad, provocando diferentes impactos. La educación es una de estas áreas, donde las posibilidades que estas tecnologías proporcionan, pueden favorecer la introducción de innovaciones en los aspectos metodológicos relacionados con los procesos de enseñanza y aprendizaje. La inserción de las TIC en los contextos educativos reporta beneficios para los alumnos, los docentes y el sistema educativo en general (Silva Quiroz, 2004).

Las TIC producen cambios en la formas de enseñanza y aprendizaje, y en el modo en que los profesores y alumnos se relacionan con el conocimiento e interactúan entre sí. Las instancias de comunicación permiten mantener un flujo de información entre el profesor y los participantes, y entre estos últimos, que les facilita poner en común ideas, compartir, reflexionar, desarrollar trabajos de carácter cooperativo y/o colaborativo, recibir retroalimentación y orientaciones por parte del tutor (Silva Quiroz, 2004).

El aula virtual es el medio en la Web en el cual los educadores y educandos se encuentran para realizar actividades que conducen al aprendizaje. Este no debe ser sólo un mecanismo para la distribución de la información, sino que debe ser un sistema donde las actividades involucradas en el proceso de aprendizaje puedan tomar lugar, es decir que debe permitir interactividad, comunicación, aplicación de los conocimientos y evaluación.

La utilización de entornos virtuales en el proceso de enseñanza-aprendizaje, en particular de la Informática, posibilita acercar al alumno a un modo de trabajo relacionado directamente con las tecnologías propias de la disciplina. De este modo, la interacción con las mismas "deja un residuo cognitivo que incorpora en los alumnos habilidades y estrategias de pensamiento que reorganizan y aumentan su rendimiento" (Madoz y Gorga, 2006).

En la Facultad de Ciencias Exactas y Naturales y Agrimensura de la Universidad Nacional del Nordeste (FACENA-UNNE), la modalidad presencial de la asignatura "Algoritmos y Estructuras de Datos I" de la carrera Licenciatura en Sistemas de Información (LSI), permite impartir conceptos teóricos básicos, cuya comprensión se refuerza luego con la implementación práctica en las clases y en los laboratorios de Informática. Esta asignatura se propone como objetivos fundamentales consolidar el pensamiento lógico que se requiere para los procesos de abstracción que demanda la programación de computadoras e introducir a los alumnos en los conceptos básicos de la Informática. Asimismo, dada su ubicación inicial en el plan de estudios, pretende apoyar activamente en el proceso de adaptación de los alumnos a las exigencias del estudio universitario.

En este trabajo se describe la experiencia de implementación de una modalidad de aprendizaje mixto o b-learning en la asignatura "Algoritmos y Estructuras de Datos I", 
mediante la incorporación de un aula virtual, la cual tiene como objetivos que los alumnos logren:

- Acceder a información de la asignatura (programa, regímenes de promoción, cronograma de clases, exámenes y otras actividades de la asignatura, difusión de calificaciones, etc.).

- Disponer del material digitalizado correspondiente a los contenidos conceptuales del programa de la asignatura, así como de los trabajos prácticos, guías de Laboratorios y software de libre distribución, que se requieren para el desarrollo de las actividades previstas.

- Informarse de novedades del mundo académico y acceder a links de sitios de interés relacionados con la asignatura.

- Interactuar con los docentes para plantear inquietudes respecto de la enseñanza o de otras cuestiones relacionadas con aspectos administrativos.

- Adquirir la habilidad para interactuar con una plataforma educativa que los motive para el desarrollo de otras propuestas de formación mediadas por la tecnología.

\section{DESCRIPCIÓN DE LA ASIGNATURA “ALGORITMOS Y ESTRUCTURAS DE DATOS I"}

La asignatura "Algoritmos y Estructuras de Datos I" pertenece al Plan de estudios 2009 de la carrera LSI de la FACENA-UNNE. Es de tipo obligatoria y se dicta en el primer cuatrimestre del primer año de la mencionada carrera. La carga horaria es de 8 horas reloj semanales, y 128 horas totales en el cuatrimestre.

Los objetivos generales de la asignatura son:

- Introducir al alumno en la programación de computadoras a través de la resolución de problemas utilizando técnicas de confección de algoritmos y codificación en un lenguaje de programación estructurado.

- Estimular en los alumnos el razonamiento lógico necesario para la resolución de problemas en el contexto del paradigma procedural o programación estructurada.

- Proporcionar los conocimientos básicos de la disciplina Informática, principalmente en los aspectos referidos al hardware y el software.

- Familiarizar a los alumnos en el uso de Internet, poniendo a su disposición toda la información necesaria en la plataforma educativa y además, ofrecer un medio de comunicación para recibir de ellos, consultas, opiniones e inquietudes.

La asignatura tiene normalmente un número elevado de alumnos cursantes, entre 300 y 350, que se agrupan en 5 comisiones para las clases prácticas. Cada comisión, a su vez, 
organiza grupos de número más reducido para las clases de laboratorio. El perfil de los alumnos es variado:

- Alumnos ingresantes a la carrera: Representan a la mayoría. Son alumnos recientemente egresados del nivel medio (17-19 años), que provienen de distintos colegios de la región y están en un proceso de ambientación al nuevo estilo de vida universitaria. Esta materia es el primer contacto con temas disciplinares específicos dentro de la carrera.

- Alumnos provenientes de otras carreras universitarias y alumnos recursantes: Son jóvenes adultos (19-21 años). Los que provienen de otras carreras universitarias están en un proceso de adaptación a la misma, los recursantes son aquellos que por diversos motivos no aprobaron la asignatura y deben volver a cursar.

- Adultos mayores: Poseen varios años de egresados del nivel medio, y además de estudiar, en general también trabajan.

Las dificultades más notorias de los alumnos son: escasa adaptación a las exigencias de la vida universitaria, ineficientes hábitos de estudio, escasa valoración del esfuerzo que se requiere para enfrentar las obligaciones académicas, nivel de conocimientos previos débiles en matemática y razonamiento lógico, poco apego a la lectura. Como contrapartida, se cuenta a favor el entusiasmo y las expectativas que tienen al inicio de la vida universitaria.

Teniendo en cuenta las características de la Informática como disciplina y la importancia creciente del software en la sociedad, desde esta materia introductoria de primer año de la carrera LSI, se propone brindar al alumno:

- Principios fundamentales de la Informática que sirvan de sustento a los conceptos y prácticas que los alumnos incorporarán gradualmente en la carrera, destacando los conceptos básicos y la terminología asociada.

- Técnicas orientadas a la resolución de problemas utilizando una computadora, incorporando el concepto de modelo como representación del ambiente, los datos y los procesos del mundo real y de la noción de programa como expresión ordenada, completa y correcta de la especificación de una solución (algoritmo) computable de dicho problema.

- Conceptos elementales para entender el funcionamiento de una computadora desde la descripción de los componentes del hardware que intervienen en el procesamiento de los datos.

- Descripción de las distintas categorías del software y sus características distintivas, brindando especial importancia a los lenguajes y paradigmas de programación vigentes.

- Síntesis sobre los temas que estudia la Ingeniería de Software, la importancia de las metodologías de desarrollo y un resumen de las "buenas prácticas" que se consideran relevantes en la construcción de software de calidad, pretendiendo que este 
conocimiento sea transversal a lo largo de su formación y que sirva de nexo entre esta materia y las subsiguientes del área Programación.

Por lo tanto, en esta asignatura se plantean dos objetivos fundamentales:

- Dar una visión general de los aspectos básicos de la Informática para que los alumnos puedan incorporar las nociones de hardware y software.

- Proporcionar conocimientos de diseño de algoritmos y programación, de tal manera que los alumnos asimilen los conceptos básicos de la resolución de problemas mediante computadoras, y adquieran la base necesaria para desarrollar programas sencillos con una metodología apropiada.

En concordancia con estos dos objetivos principales de la asignatura, los contenidos de la misma se encuentran estructurados en dos partes: "Conceptos Fundamentales" y "Algoritmos y programación", tal como puede observarse en el siguiente mapa conceptual de la asignatura (Figura 1).

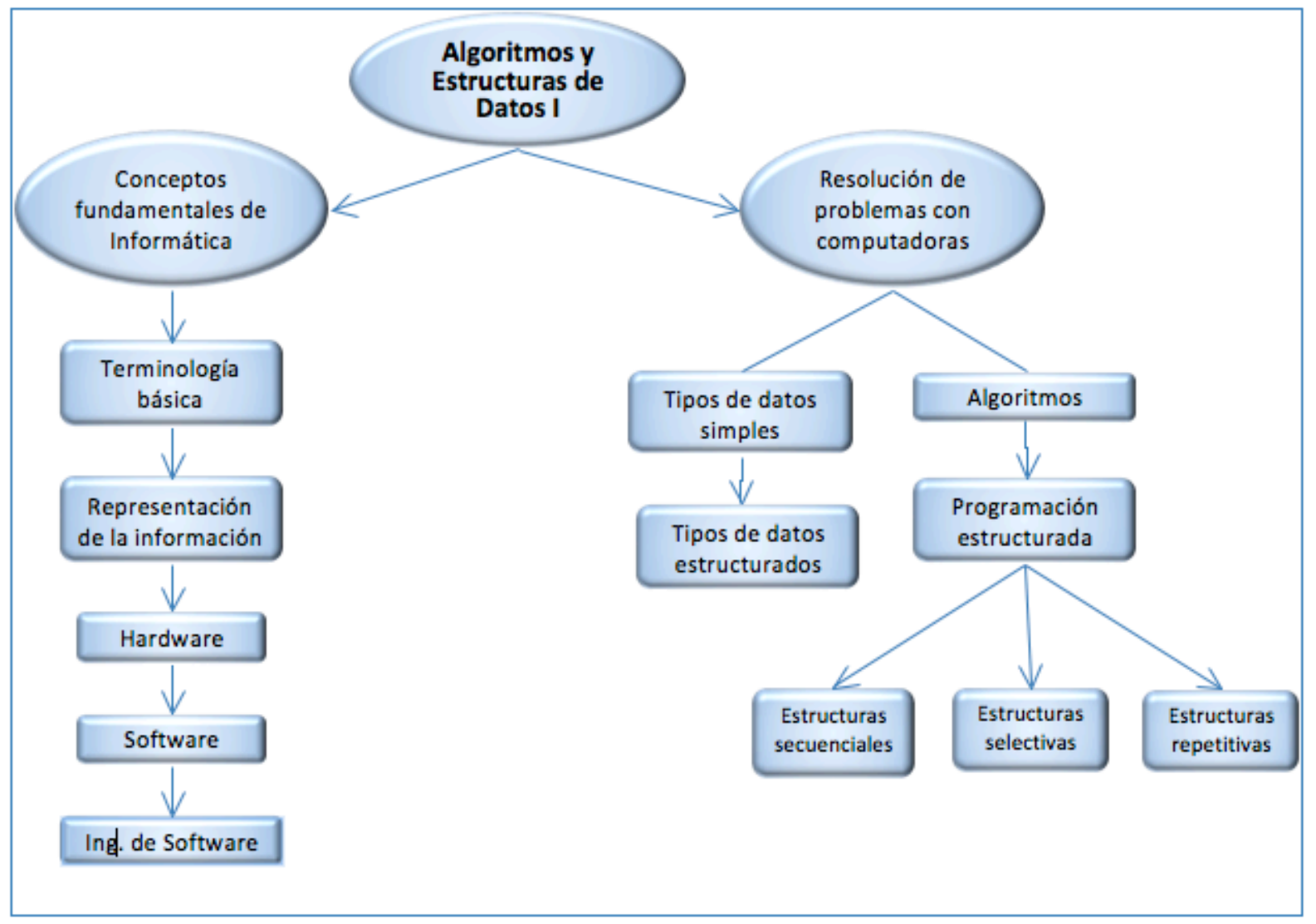

Figura 1. Mapa conceptual de la asignatura "Algoritmos y Estructuras de Datos I" 


\section{EXPERIENCIA DE IMPLEMENTACIÓN DE UN AULA VIRTUAL ORIENTADO A UNA MODALIDAD B-LEARNING}

Se describe la metodología que implementa un aula virtual como apoyo a las actividades presenciales de la asignatura Algoritmos y Estructuras de Datos I, orientada a una modalidad de aprendizaje mixto o b-learning.

Al hablar de "b-learning o blended learning", se hace referencia a la combinación de enseñanza presencial con tecnologías para la enseñanza a distancia, o a aquellos procesos de aprendizaje realizados a través de los sistemas y redes digitales, pero en los que se establecen una serie de sesiones presenciales o situaciones que propician el contacto cara a cara (García Aretio, 2007).

Los sistemas basados en el modelo b-learning, como aulas virtuales para el apoyo de la formación presencial, se caracterizan por la flexibilidad e interactividad que facilitan los recursos de información y las herramientas comunicativas (chat, correo, foros de discusión, weblogs, sistemas de mensajería instantánea), lo cual permite superar obstáculos en el desarrollo de trabajos en grupo y búsqueda de respuestas y soluciones conjuntas entre los alumnos, propios de los sistemas presenciales, tales como la limitación espacio-temporal de las reuniones de los grupos de trabajo, el no poder compartir la información simultáneamente, la limitación de la información a utilizar, entre otros (Sosa Sánchez-Cortés et al., 2005).

Este modelo de tipo mixto que combina los mejores recursos de la ofertas educativas presenciales y las realizadas en una modalidad a distancia, ha demostrado ser la tendencia actual, debido a la posibilidad para los docentes de analizar la mejor propuesta didáctica con incorporación de todos los recursos de acuerdo a los destinatarios, contexto y temática a abordar o habilidad a desarrollar en los alumnos (Sanz et al, 2006) citado en (Mariño y López, 2007).

Marsh (2003) citado en Cataldi et al (2005) menciona dos estrategias que tratan de mejorar la calidad con "blended learning": una es otorgar más responsabilidad a los estudiantes en su estudio individual proporcionándoles destrezas para dicho estudio, y la otra es mejorar la calidad de las clases mediante el uso de presentaciones Multimedia.

El modelo de aprendizaje combinado o b-learning implementado en la asignatura está caracterizado por:

- Clases teóricas (obligatorias): Brindan los conceptos necesarios que serán integrados con las actividades prácticas. Se desarrollan a través de exposiciones con el apoyo de diapositivas presentadas mediante proyector multimedia. Al final de cada tema, se 
brinda un cuestionario que los alumnos deben resolver en forma grupal o individual con el objeto de consolidar los conceptos relevantes.

- Clases prácticas (obligatorias): Tienen como propósito fundamental que los alumnos consoliden el pensamiento lógico y la capacidad de abstracción que se requiere para resolver problemas utilizando computadoras. En las mismas los alumnos resuelven los ejercicios propuestos en guías de trabajos prácticos, elaboradas con el propósito de afianzar los conceptos teóricos y estimular la capacidad de resolución de problemas, las cuales pueden ser resueltas en forma individual o grupal. Durante las clases prácticas los docentes cumplen la función de guía-consultor, respondiendo a las cuestiones planteadas por los alumnos.

- Clases de laboratorio (obligatorias): El objetivo específico de estas clases es que los alumnos completen la visión del "ciclo de vida del desarrollo del software", realizando todas las etapas del mismo: análisis, diseño, codificación, ejecución y prueba del programa, de manera que puedan consolidar su capacidad para resolver problemas mediante la programación. El trabajo de los alumnos es orientado mediante Guías de laboratorio, las cuales pueden ser desarrolladas en forma individual o grupal. En esta instancia también los docentes cumplen la función de guía-consultor, mediante la atención de consultas y el apoyo necesario para que el alumno supere las dificultades que se presenten.

- Repositorio actualizado de material didáctico: La asignatura dispone de material didáctico consistente en apuntes teóricos de todos los temas del programa, las guías de trabajos prácticos que se desarrollan en las clases prácticas y las guías de trabajo que se realizan en el Laboratorio de Informática. Además, se ha desarrollado material multimedial para cada uno de los temas del programa de la asignatura, utilizando software de diseño de presentaciones. Todo el material mencionado se encuentra disponible en la sección Materiales del aula virtual de la asignatura.

- Comunicación sincrónica y asincrónica: Las herramientas de comunicación sincrónica (chat) y asincrónicas (mensajería) disponibles en el aula virtual, son los medios empleados para atender las consultas surgidas del estudio independiente con apoyo del material didáctico.

- Estudio independiente: Los alumnos pueden acceder al material disponible sin restricciones espacio-temporales. Las consultas y comunicaciones se mediatizan entre los estudiantes entre sí o entre ellos y con los docentes. Por otra parte, se ofrecen guías de trabajos prácticos adicionales, con una amplia variedad de ejercicios para que los alumnos las desarrollen fuera del horario de clase, con el objeto de estimular la capacidad de resolución de problemas y el estudio independiente. 
La asignatura se dicta en el primer cuatrimestre del año, desde marzo hasta finales a junio. Al inicio del cursado, se pone a disposición de los alumnos en el aula virtual un cronograma de todas las actividades previstas en la asignatura: clases teóricas, prácticas y de laboratorios, exámenes parciales y finales. Esta información tiene como objetivo que el alumno organice apropiadamente su tiempo de estudio y defina su propia planificación o estrategia de cursado, en función de las otras obligaciones académicas.

Este aspecto, la administración del tiempo de estudio, se observa como crítico en los alumnos dado que, por su condición de ingresantes, muchas veces se encuentran desbordados en su capacidad de atender las exigencias de las distintas asignaturas. Por lo tanto, anticipar estas situaciones puede resultar beneficioso para su desempeño.

En el segundo cuatrimestre, el aula virtual permite mantener la vinculación con los alumnos, una vez finalizado el cursado. Aprovechando esta facilidad, la asignatura implementa un plan especial de apoyo a los alumnos que no han logrado cumplimentar satisfactoriamente los requisitos de aprobación de la materia. Éste consiste en un conjunto de actividades especialmente diseñadas para favorecer la comprensión de los temas que resultan más dificultosos a los alumnos. Las actividades se desarrollan a través del aula virtual, en la cual cada docente tutor, asignado a un reducido número de alumnos (no más de 10), monitorea el cumplimiento de las consignas por parte de los mismos y apoya su aprendizaje con un seguimiento personalizado. Se espera que esta preparación facilite a los alumnos aprobar la asignatura en las instancias de exámenes finales previstos en el calendario académico.

En relación a la evaluación del aprendizaje, los criterios son los siguientes:

- Capacidad para resolver los problemas planteados utilizando las técnicas de la programación estructurada.

- Capacidad de abstracción y de modelización.

- Utilización de los términos técnicos específicos.

- Capacidad de síntesis y de relación de los distintos conceptos.

Según Palou de Maté (2003), la evaluación tiene dos funciones: la evaluación de los procesos de aprendizajes y la evaluación de resultados, con distintas finalidades: la de realimentar el proceso de enseñanza, en el primer caso, y la de acreditación, en el otro. La evaluación alternativa, como la denominan diversos autores, focaliza la evaluación sobre el alumno, según sus propios aprendizajes, habilita al evaluador a crear una historia evaluativa respecto del individuo o del grupo, provee información que facilita la acción curricular, y permite a los estudiantes participar en su propia evaluación (Mateo, 2000). 
En la asignatura se implementan diferentes mecanismos e instrumentos de evaluación que permiten obtener información sobre los logros alcanzados por los alumnos durante su aprendizaje y evidenciar los aspectos de la docencia que puedan ser susceptibles de mejora. A continuación, se describen los instrumentos que se implementan en la actualidad:

- Exámenes parciales escritos teórico-prácticos: Incluyen resolución de ejercicios y problemas, y cuestionarios con preguntas de respuesta abierta y preguntas de respuesta múltiple.

- Exámenes finales escritos (parte práctica) y orales (teoría): las fechas de las mesas de exámenes de la asignatura se encuentran consignadas en el calendario de exámenes de la FACENA-UNNE.

- Trabajos prácticos grupales obligatorios: Los alumnos deben desarrollar dos trabajos prácticos que consisten en la resolución de un problema mediante la técnica de seudocódigo y su posterior codificación en un lenguaje de programación procedural. Los trabajos deben enviarse a través del aula virtual al docente responsable de la comisión a la que pertenece el alumno. La consigna de presentación de estos trabajos define protocolos, tanto para la elaboración como para el envío del trabajo, debiéndose respetar un formato para el asunto del mail, y para el nombre del archivo que se adjunta. El propósito de esta actividad es introducir al alumno a las buenas prácticas sobre el intercambio de información de manera virtual, sujeto a reglas y protocolos, fomentando así el trabajo profesional.

Asimismo, con el objeto de recabar información para evaluar y retroalimentar la metodología de enseñanza, se aplican dos encuestas, una al inicio y la otra al final del cursado:

- Encuesta Inicial: El objetivo es obtener información acerca del perfil de los alumnos, sus conocimientos previos, motivación de ingreso a la carrera, etc., que aporta un mayor conocimiento de los mismos.

- Encuesta Final: El objetivo es evaluar el grado de satisfacción de los estudiantes en relación a la asignatura y al uso del aula virtual. Los resultados obtenidos en este relevamiento permiten obtener información para mejorar en el futuro la metodología que integra el aula virtual como recurso complementario en el proceso de afianzamiento de conocimientos impartidos, y además redefinir las estrategias de dictado de las clases teóricas y prácticas, atendiendo particularmente a las características de los alumnos que cursan la misma. 


\section{Propuestas futuras:}

Para consolidar los conceptos de la asignatura, se propone completar la metodología de enseñanza-aprendizaje, aprovechando el aula virtual disponible, mediante las siguientes actividades a incorporar a futuro:

- Actividades de autoevaluación: Permiten a los alumnos realizar un seguimiento de su aprendizaje de forma autónoma y libre y, a su vez, posibilitan aprender de sus errores. "Nadie mejor que el sujeto que aprende para conocer lo que realmente sabe. Autoevaluarse implica tomar nota de la marcha del propio aprendizaje. La evaluación debería tener siempre como meta final la autoevaluación, ya que debe propiciar en el sujeto la autoconciencia de sus procesos de aprender" (Sanjurjo et.al.,1998).

Las actividades de autoevaluación estarán disponibles en el aula virtual, y podrán ser de tipo crucigramas, preguntas de selección de alternativas múltiples o ejercicios de emparejamiento y de rellenar huecos, para el afianzamiento de diferentes temas del programa.

- Webquest: Para guiar el proceso de indagación e investigación de los alumnos, especialmente en la Web, se propone la utilización de la metodología Webquest, evitando de este modo la navegación simple y muchas veces sin rumbo de los mismos a través de la Web, y para reducir el tiempo empleado en encontrar la información solicitada. Esto posibilita que los alumnos empleen ese tiempo en analizar la información, comprenderla, elaborar resúmenes o cuadros comparativos, redactar conclusiones propias, etc. Se solicitará la elaboración de un Webquest para los temas Hardware de procesamiento de datos y Software y lenguajes de programación. Los alumnos podrán optar por cualquiera de ellos, estando estas actividades disponibles en el aula virtual.

- Foros: Esta sección prevé la realización de dos tipos de actividades: Una, orientada a recabar la opinión de los alumnos acerca de sus dificultades en el uso de las herramientas propuestas, la comprensión de enunciados y consignas de trabajo, etc. Otra, orientada a favorecer la integración de los alumnos mediante el desarrollo de trabajos grupales colaborativos, consolidando el trabajo en equipo, competencia especialmente requerida en los profesionales de la disciplina.

La participación de los alumnos en estas actividades será obligatoria, y se le asignará una nota conceptual.

Por otra parte, mediante el análisis de la información que provee el aula virtual, se evaluará la actividad de los alumnos en el mismo, a través de los siguientes elementos:

- Frecuencia de acceso al aula virtual (por día, semana, mes ó por secciones del aula). 
- Descarga del material didáctico disponible en las distintas secciones del aula.

- Desarrollo de las actividades de autoevaluación diseñadas para cada tema.

- Participación en los Foros abiertos.

- Realización y presentación de los trabajos grupales colaborativos propuestos a través de foros.

- Utilización de la mensajería interna (correo electrónico) y el chat disponibles en el aula virtual.

- Uso de un lenguaje adecuado al contexto (actitudes de respeto y compañerismo) en los foros, chat y correo electrónico.

\section{SISTEMA TUTORIAL}

Para la implementación del modelo de aprendizaje combinado o blended learning, descripto en la sección 3, se utilizó el aula virtual habilitada para la asignatura en la plataforma tecnológica disponible en la institución (UNNE Virtual, 2004). El aula dispone de las siguientes opciones en sus diferentes secciones:

\section{- Sección Materiales:}

o Subsección Contenidos: A su vez se divide en las siguientes subsecciones:

- Condiciones y contenidos: Permite acceder al régimen de regularización y promoción de la asignatura, al programa analítico, y a la planificación de las actividades previstas para el cuatrimestre.

- Teoría: Permite la descarga de los módulos de teoría correspondientes a los temas del programa de la asignatura.

- Prácticos: Permite la descarga de las Guías de Trabajos Prácticos y de Guías de ejercicios complementarios.

- Laboratorio: Permite la descarga de las Guías de Laboratorio.

o Subsección Calificaciones: Publicación de calificaciones obtenidas por los alumnos en los exámenes parciales, finales y trabajos prácticos.

o Subsección Noticias: Presentación de las novedades referentes a fechas de exámenes, actualización o incorporación de nuevo material didáctico, etc.

o Subsección Links de interés: Lista de enlaces a sitios web recomendados por los docentes de la asignatura. 


\section{- Sección Interacción:}

o Subsección Calendario: Publicación del cronograma de actividades programadas para el cuatrimestre. Por ejemplo: clases teóricas, prácticas y de laboratorio, fechas de exámenes parciales y finales, fechas de presentación de trabajos prácticos, conferencias, congresos, etc.

o Subsección Chat: permite la evacuación de dudas de los alumnos mediante el intercambio en tiempo real con los docentes-tutores. El uso de este medio de comunicación sincrónica es opcional.

o Subsección Contactos: Se proporciona una dirección de e-mail para facilitar las consultas de los estudiantes a los docentes-tutores, ya sean de carácter administrativo o generales acerca de la asignatura. El uso de este medio de comunicación asincrónica es opcional.

o Subsección Mensajería Interna: Permite la comunicación asincrónica de los docentestutores con los alumnos con diferentes objetivos: reforzar información publicada en plataforma, responder consultas, solicitar información, invitarlos a participar de los foros u otras actividades, etc. El uso de este medio de comunicación es obligatorio en algunos casos (envío a los docentes-tutores de trabajos de presentación obligatoria), y opcional en otros.

o Subsección Consultas Frecuentes: Publicación de respuestas a temas operativos (qué estudiar para un final, cómo es el examen de un alumno libre, etc.) o respecto de contenidos específicos de la asignatura que los alumnos planteen asiduamente.

La función tutorial, desde el punto de vista de la enseñanza, consiste en potenciar la actividad del estudiante según su propio proceso de aprendizaje, ofreciéndole herramientas apropiadas que estimulen su autonomía, y orienten y faciliten su aprendizaje dotándolo de significatividad. Para un eficiente funcionamiento del aula virtual, es fundamental el desempeño de los docentes coordinadores y los docentes tutores, quienes tienen la misión de oficiar de mediadores entre los alumnos y las herramientas tecnológicas.

El equipo de docentes a cargo del aula virtual se compone de la siguiente manera:

- Docentes Coordinadores, quienes llevan a cabo las siguientes tareas en el ámbito del aula virtual, además de las tareas que les son inherentes a sus funciones en la modalidad presencial.

o Orientación de la organización, el estilo y la implementación del aula virtual de la asignatura. 
o Planificación, coordinación y supervisión de las tareas del equipo de docentes tutores.

o Colocación en la plataforma virtual del material didáctico de la asignatura.

o Administración de la sección Noticias en la que se difunden las novedades de la asignatura.

o Resolución de los problemas que puedan surgir en relación al aula virtual.

o Publicación de información sobre actividades que puedan ser de interés para los alumnos: cursos, conferencias, etc.

- Docentes Tutores, quienes llevan a cabo las siguientes tareas en el ámbito del aula virtual, además de las tareas que les son inherentes a sus funciones en la modalidad presencial

o Corrección de los trabajos prácticos y devolución de las calificaciones a los alumnos.

- Atención de las consultas de los alumnos realizadas a través de los medios de comunicación sincrónica (chat) y asincrónica (mensajería). Se considera primordial contestar a todas las inquietudes que los alumnos tengan, ya sean académicas o tecnológicas, y realizar al mismo tiempo un acompañamiento, para evitar el abandono del cursado de la asignatura. Asimismo, realizar una devolución rápida de las inquietudes, permite sostener el aprendizaje autónomo que realizan los estudiantes. Los docentes tutores deberán informar a los alumnos los horarios en los cuales harán la atención en línea de las consultas a través del Chat.

o Realización del seguimiento de las actividades por parte de los alumnos, verificando si envían los trabajos prácticos obligatorios en tiempo y forma.

o Detección de distintas problemáticas que puedan surgir en relación al aula virtual, y comunicación de las mismas a los docentes coordinadores (por ejemplo, uso de lenguaje inapropiado, etc).

\section{CONCLUSIONES}

Se ha presentado una experiencia de implementación de una metodología de enseñanza aprendizaje en la asignatura "Algoritmos y Estructuras de Datos I", de la carrera LSI de la FACENA-UNNE, a través de la incorporación de un aula virtual haciendo uso de la plataforma UNNE Virtual.

La misma constituye una herramienta apropiada para mejorar la comunicación con los alumnos, poniendo a su disposición todo el material didáctico y software que se requieren para el desarrollo de las actividades, a la vez que permite a los docentes atender las 
consultas e inquietudes, logrando de este modo un acercamiento a las ventajas de las TIC y contribuyendo a mejorar la calidad de la formación.

La modalidad propuesta contribuye a ampliar el manejo de herramientas informáticas por parte de los alumnos. A su vez, favorece la comprensión del concepto de software de aplicación en estudiantes que están iniciándose en la adquisición de técnicas de desarrollo de software.

Asimismo, el entorno tecnológico posibilita una amplia variedad de recursos orientados a consolidar los conceptos, tales como la autoevaluación, técnicas de webquest, etc., que a futuro se incorporarán al aula virtual enriqueciendo la metodología de enseñanza, lo que implica para los docentes un desafío para repensar las prácticas en función de los recursos disponibles.

Finalmente, se considera de gran importancia la formación de los docentes universitarios en educación virtual para permitir la implantación de los entornos virtuales de aprendizaje en las asignaturas de carreras de grado y posgrado, así como también la preparación, motivación y apoyo a los alumnos en el uso de los mencionados entornos virtuales.

\section{REFERENCIAS}

CATALDI, Z., FIGUEROA, N., LAGE, F., GRAUS, G., BRITOS, P., GARCÍA MARTÍNEZ, R. (2005). EI rol del profesor en la modalidad de b-learning tutorial. http://www.itba.edu.ar/capis/webcapis/RGMITBA/comunicacionesrgm/CIESyNT-2005T192.pdf.

GARCÍA ARETIO L., RUIZ CORBELLA M., DOMÍNGUEZ FIGAREDO D. (2007). De la educación a distancia a la educación virtual. Edit. Ariel. ISBN978-84-344-2666-5.

MADOZ, C., GORGA, G. (2006). Análisis del proceso de articulación para alumnos de Informática, utilizando herramientas de Educación a Distancia. Revista Iberoamericana de Tecnología en Educación y Educación en Tecnología (TE\&ET). Vol. 1, Nro. 1. 2006.

MARIÑO, S. I., LÓPEZ, M. V. (2007). Aplicación del modelo b-learning en la asignatura 'Modelos y Simulación' de las carreras de Sistemas de la FACENA- UNNE. Edutec: Revista Electrónica de Tecnología Educativa. ISSN: 1135-9250. N²3. Julio de 2007. España. 14 pgs. http://edutec.rediris.es/Revelec2/revelec23/revelec23.html

MATEO, J.A. (2000). La evaluación educativa, su práctica y otras metáforas. ICE, Universidad de Barcelona. España. 
PALOU DE MATÉ, C. (2003). La enseñanza y la evaluación. Una propuesta para Matemática y Lengua. Universidad Nacional del Comahue. Cipolletti, Río Negro. Argentina.

SANJURJO, L., VERA, M. (1998). Aprendizaje significativo y enseñanza en los niveles medios y superior. Homo Sapiens. Buenos Aires. Argentina.

SILVA QUIROZ, J. E. (2004). El rol del tutor en un ambiente virtual de aprendizaje para la formación continua de docentes. Revista Teoría de la Educación en la Sociedad de la Información, 2004, Vol. 5. Ediciones Universidad de Salamanca. España.

SOSA SÁNCHEZ-CORTÉS, R., GARCÍA MANSO, A., SÁNCHEZ ALLENDE, J., MORENO DÍAZ, P. Y REINOSO PEINADO, A. J. (2005). B-Learning y teoría del aprendizaje constructivista en las disciplinas Informáticas: un esquema de ejemplo a aplicar. Recent Research Developments in Learning Technologies.

UNNE-VIRTUAL (2004). LINEAMIENTOS DE ACCION. Res.451/04 CS. Universidad Nacional del Nordeste. http://virtual.unne.edu.ar/institucional/resoluciones.php

Para citar este artículo:

LÓPEZ, M.V., DAPOZO, G.N., GREINER, C.L. \& ESPÍNDOLA, M.C. (2012). Entorno virtual como soporte a la formación de informáticos en una Universidad del Nordeste Argentino. EDUTEC, Revista Electrónica de Tecnología Educativa, 39. Recuperado el dd/mm/aa de http://edutec.rediris.es/Revelec2/Revelec39/entorno virtual soporte formacion informati cos universidad.html 